20

\title{
Broadband intelligent network project
}

\author{
Olli Martikainen, \\ Telecom Finland, \\ P.O.BOX 106, 00511 Helsinki \\ Tel: +358 20403513 , Fax: +35820403251
}

Kim Molin,

Lappeenranta University of Technology,

Telecommunications laboratory,

Laserkatu 6, 53850 Lappeenranta

Fax: +358 53574 3650,

E-mail: Kim.Molin@lut.fi

\begin{abstract}
This paper presents a case study on a service architecture called Broadband Intelligent Network $(B I N)$. BIN consists of Broadband Service Subscribers (BSS), Broadband Service Switching Point (BSSP or ATM-switch), Broadband Service Control Point $(B S C P)$, Broadband Service Management Point (BSMP) and Broadband Service Providers (BSP).

Unlike in conventional IN, BIN service logic programs are distributed into each active component of BIN. BSS and BSCP contain the basic service logic programs with the basic functions such as controlling the icons, the hypermedia documents, QoS and charging. In the project a sophisticated application protocol was designed, called BINAP (Broadband INAP). BINAP is ends-oriented being used as an application protocol between the components of BIN. BINAP includes messages for security, performance, Quality of Service $(Q o S)$, managing customer service palettes and controlling the multimedia flow between the BSS, BSCP and BSP.

Services provided in BIN are based on the transfer of multimedia data from BSP to BSS, known also as the fast multimedia data stream. The control of the services is provided by BSCP together with the distributed service logic.
\end{abstract}

Keywords: ATM, Broadband ISDN, BIN, IN, TMN 


\section{INTRODUCTION}

Telecom Finland and Lappeenranta University of Technology (LUT) have started a project to research broadband, ATM-based, Intelligent Network called the Broadband Intelligent Network $(B I N)$. The research considers the future broadband multimedia services and their implementation. BIN has not been standardized or even implemented anywhere, being now in the prototype design phase. It has been only a name for the project, which was started in March 1994 at Telecom Finland /4/.

The project refers to the conventional narrowband IN /1/ with fast service introduction and support for external broadband service providers. The main objectives concerning the architecture have been centralized customer and service management and multiservice offerings at single service points.

\section{BIN ARCHITECTURE}

\subsection{Components}

BIN components are BSSs, BSPs, BSCPs, BSMPs and BSSPs (Figure 1). BSSs, BSPs and BSCPs are connected to BSSPs, which form the ATM-network. BSMP is connected to BSCP and is not tied to any physical implementation technique.

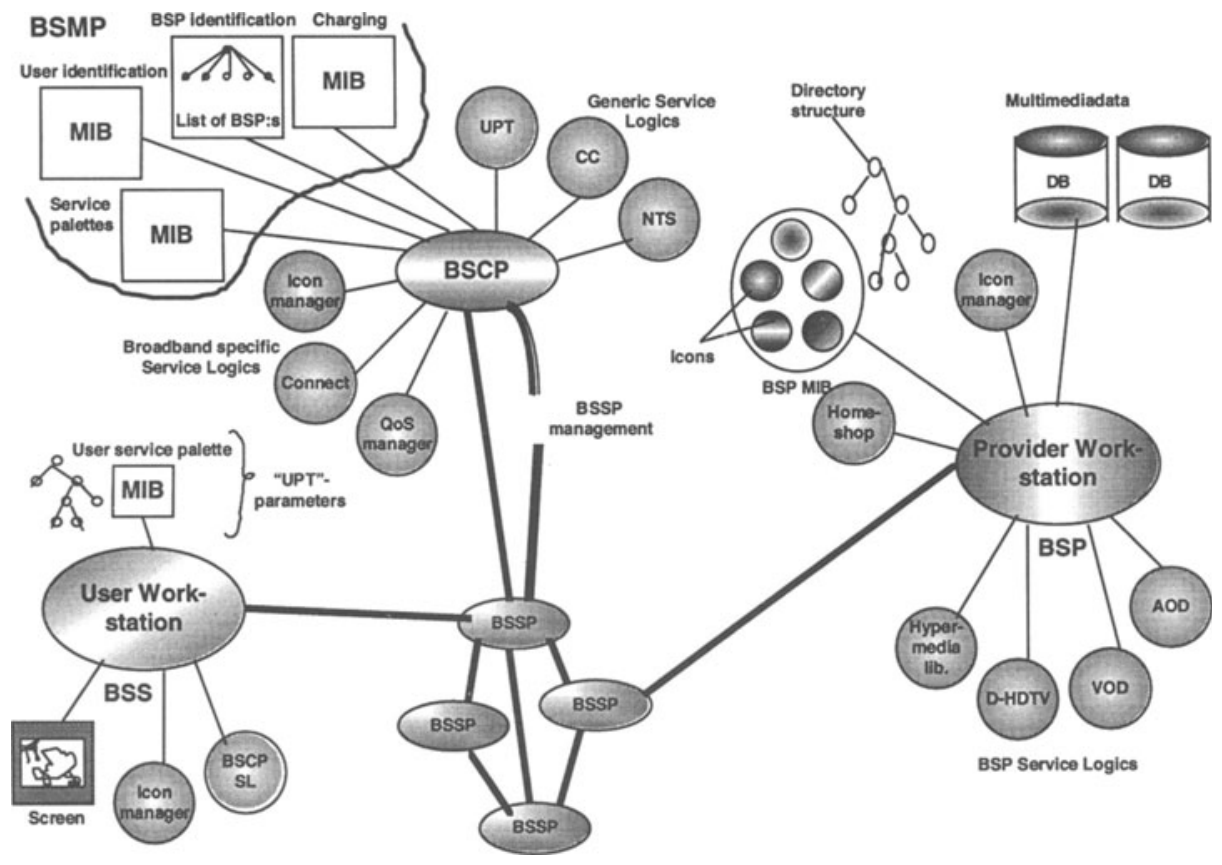

Figure 1. BIN Architecture. 
Several service providers, which would use different protocols and managing architecture, could be designed. If done this way, every service provider should implement its own mechanisms of charging and managing services. One advantage of this architecture would be quite simple interface to the end user, and one disadvantage quite complex service introduction. Intelligent Networks have centralized service management architecture, whereby components can be distributed from each other. The objective of this project is to have a centralized model, where the users (BSS) could use services provided by BSPs and have a centralized service management system.

BSMP contains customer identification and charging information, a list of BSPs known to it and possibly a customer service palette. BSCP contains the general Service Logics $(S L)$ also existing in conventional IN, which can be used in several services, such as CC (Credit Calling), NTS (Number Translation Service), and UPT (Universal Personal Telecommunications). In addition, broadband services have SLs for controlling icons and QoS (Quality of Service) -parameters, and SL for a connection initializer. BSS has possibly its own customer service palette, icon manager, SL for BSCP and a screen handler for showing the multimedia data. In BSP lies the actual multimedia databases $(D B)$ and icon databases. Also the SLs for broadband services are located there.

\subsection{BIN and IN}

In conventional narrowband IN the user has a simple interface to the network, i.e. either from the SSP (Service Switching Point) or the NAP (Network Access Point). The highly simplified function of CS1 (Capability Set 1) IN is that the user is connected via SSP or NAP to the SS7 (Signalling System No. 7) network which forms the signalling network of the IN. The user dials a phone number, which is then generated into a message and transferred to the nearest SSP. If the number begins with a 0700 or 0800 , SSP knows that the user chose an IN number. In this case, SSP triggers an IN call, otherwise the call setup procedure is just as for a normal call. SSP forms the intelligent part of conventional IN. SSP triggers the IN call and forwards an INAP (Intelligent Network Application Protocol) message to the SCP (Service Control Point) via the SS7 network using the services of TCAP (Transaction Capabilities Application Part). SCP then has control on the next step e.g. sends a control message to SSP.

In BIN the function of BSCP is different from SCP. BSSP is in point of fact a simple highspeed switch architecture, e.g. ATM-switch. The functions of such a switch is to route the 53byte cell from the input to output end according to the Virtual Circuit Identifiers $(V C I)$ and Virtual Path Identifiers (VPI) and the information that has been configured in its routing tables. The switch is not intelligent like conventional IN switch, because it does not trigger an IN call. It handles any data in the cells transparently. Compared to the SS7 network's $64 \mathrm{Kbit} / \mathrm{s}$ capacity ATM-network forwards cells at a much higher rate i.e. $\geq 155 \mathrm{Mbit} / \mathrm{s}$.

SSP solutions are mainly based on hardware solutions. They can not be programmed as easily as computers. While BSSP being simple, the BSCP has to be quite complex. The end nodes, BSS and BSP, are thus also quite complicated. This means that application layer protocol BINAP is tranferred between all the end nodes unlike in conventional IN, where INAP is mainly used between SSP and SCP. 


\subsection{Broadband services}

In fact the few services shown in table 1 are in a way quite similar to each other. They could be grouped into three different categories: controlled file transfer ${ }^{1}$ based AOD and VOD, hypermedia database ${ }^{2}$ based hypermedia library and homeshopping, and single- or multiparty ${ }^{3}$ calls. By doing this controlling of the services can be done in a similar way. There does not have to be different controlling mechanisms for every service provided.

Table 1. Some broadband services
AOD
(1) AOD (Audio On Demand) is a service that corresponds to a CD- (Compact Disk) player. The service includes therefore PLAY and STOP functions.
VOD VOD (Video On Demand) is similar to AOD, but in addition to voice also moving picture is transferred. In future, it might be competing with the video rent activity.

\section{Hypermedia \\ library \\ Homeshopping}
Videotelephony
Video
conferencing
2 An interactive service, where the user can browse hypermedia documents over the network, e.g. real-time Internet Mosaic. An interactive homeshopping service that is based on hypermedia documents.
(3) Videotelephony is a conventional two-party telephone, where in addition to voice also moving video picture is transferred. Videoconferencing is quite similar to videotelephony, but it enables multiparty instances.

\subsection{Functioning of BIN architecture}

The main idea of BIN architecture is that the user does not directly communicate with the service provider with BINAP messages. BSCP, which forms the controlling part of the BIN, processes the BINAP message sent to it, makes statistics, and controls other points by sending BINAP messages to them. The advantage of this kind of architecture is that the end systems (BSS and BSP) do not have to be as complex as the controlling systems. The main intelligence of a service lies in BSCP and BSP, where are the BSLs (Broadband Service Logic). BSS has mainly the intelligence of requesting a service and interpretating BINAP messages sent to it.

BIN consists of two data streams: the management data streams and multimedia data streams. BSS communicates only with BSCP, but BSCP is responsible for communicating with all the other components. The transport layer below BSS does communicate with the BSP, but it gives upwards only data indications from BSP. (Figure 2) 


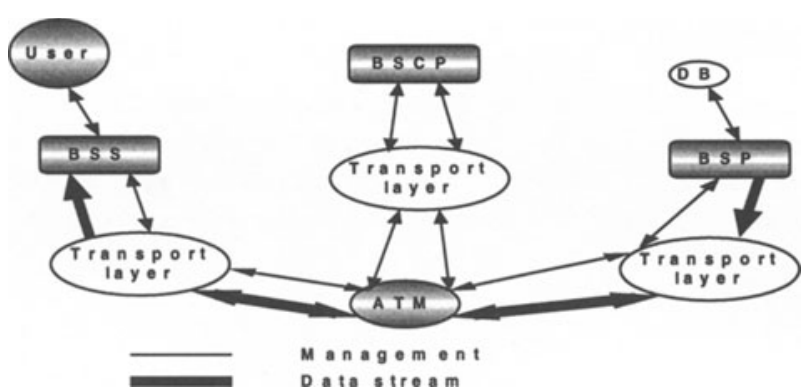

Figure 2. Management and data streams separated.

\subsection{Requirements of ATM network}

The signalling protocol itself is not yet developed to the level where it could be used to implement BIN architecture using ATM. The BSS uses the ATM signalling protocol (Q.2931) to set up the path to the destination, which is BSCP, or it could use the PVCs that are available to BSCP. However, the BIN architecture suggests to use BSCP to establish the path between BSP and BSS. The Q.2931 signalling protocol does not define these kind of functions, but similar extension as Q.932 to Q.931 in ISDN is needed /2,3/. Q.2931 UNI version 3.0 does not define a third parties connection setup. UNI 4.0, which is going to be introduced in late 1994 , should contain the third party connection setup function defined.

\subsection{Course of BIN events}

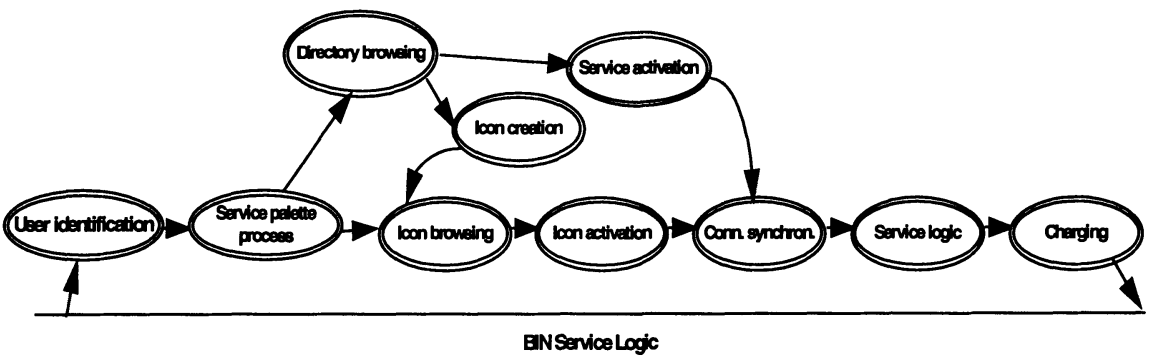

Figure 3. Course of BIN events.

Let us consider the event sequences when applying BIN services (Figure 3).

\subsubsection{Service request phase}

\section{User identification:}

The user sends a BINAP message to BSCP and gives sufficient identification information of $\mathrm{him} /$ herself. The user has to know the ATM-address (CCITT NSAP (Network Service Access Point), 20 bytes) of BSCP. BSCP then fetches more accurate information about the user, whereby the location of the customer service palette is also found. If the user information is 
not found in the current BSMP, the user must give the address of his/her Home BSCP $(H B S C P)$. This enables usage of broadband services from mobile stations.

Directory browsing:

In case of a new user, requesting of a service is proceeded via directory browsing. BSCP knows one or more BSPs. BSCP sends a BINAP message to BSS, which contains a hypermedia document with links to BSPs. (Figure 4) The first level of the hypermedia document contains the BSPs (located at BSCP) and next levels contain all the information the BSP is able to offer, which are fetched by BSCP from the BSP in case. BSCP does not have to know all the services that every BSP can offer, just knowing the addresses of the BSPs is sufficient. By using the identification information of the user, the BSCP may filter the information given to the user.

The hypermedia documents residing in BSPs contain structured information about the type of service. The types are controlled file transfer, hypermedia database or single- and multiparty calls.

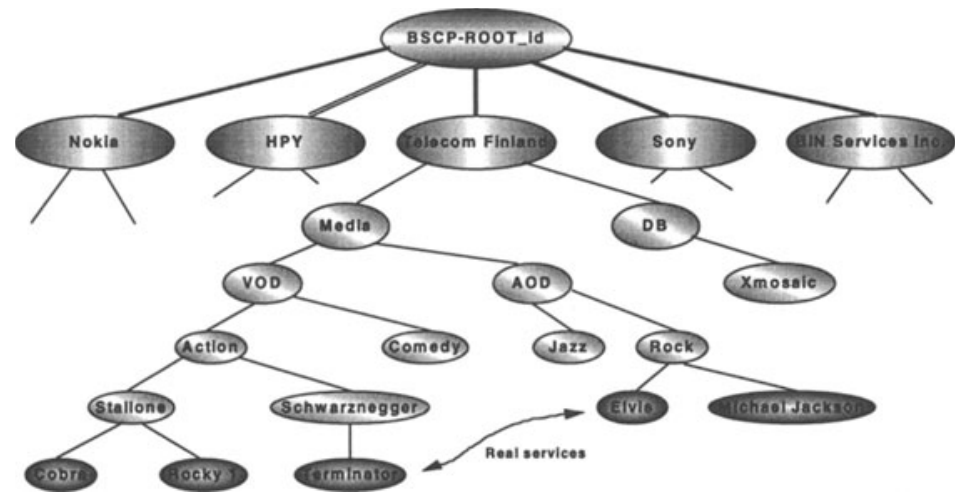

Figure 4. The structure of directory tree.

Service palette process:

When the user has chosen the 'real' service, an icon can be created to the customer service palette either to BSMP or BSS. When such a service palette exists containing icons and an accurate description of the parameters of the service, the service may be executed via icon browsing and activation. This enables much simpler and faster usage of broadband services, because of icons' graphical presentation and short-way execution process.

\subsubsection{Service activation phase}

After activation of the icon or 'real' service, BSS informs BSCP, which sends BSP a BINAP message containing sufficient information about the icon or 'real' service. In BSP the BSL for the service is executed. In BSCP the QoS-manager is initialized for this connection, which has received the QoS-parameters from the BSS. The QoS-parameters are also negotiated with BSP and if the user has too little network capacity, the BSP might reject the service usage. 


\subsubsection{Service usage phase}

This phase is highly dependent of the type of service requested. For example, controlled file transfer type of service could have the functions of PLAY and STOP. During the service execution phase the QoS-manager is responsible for the quality parameters of the service. The parties inform BSCP of the changes in service quality, which then tries to restore the values.

\subsubsection{After-usage phase}

After the service has been used, the user should inform BSCP of the connection closing. BSCP then starts the controlled connection close phase. BSCP has stored the necessary information about the service usage, e.g. actual usage time and ability to perform with the requested QoSparameters. The actual time here means for example in controlled file transfer the time between PLAY and STOP functions summed up in the entire session. The charging information is then added to the MIB (Management Information Base) in the BSMP of the user.

\section{BINAP}

\subsection{BINAP-messages}

BIN uses BINAP application protocol to communicate with the external parties of BSCP. BINAP-messages have been categorized into the following subclasses:

Initialization:

- User identification

- Customer service palette

- Directory handling

Service usage:

- Service type dependent control messages during the service usage

- QoS-messages

Service close:

- Controlled closing messages

- Customer charging

\section{CUSTOMER SERVICE PALETTE}

\subsection{BIN conceptual model}

The BIN conceptual model does not correspond to the conventional IN conceptual model and it is divided into to three planes: user, network and service planes (Figure 5). 


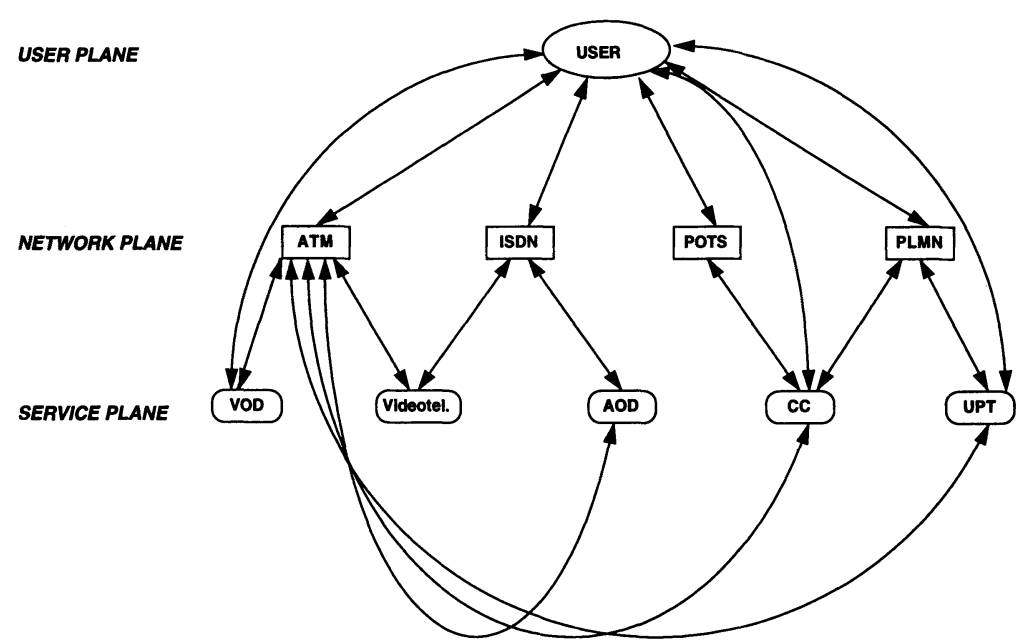

Figure 5. BIN conceptual model.

The user plane contains the information about the user. The network plane defines all the possible networks that the user system can interface with. The service plane indicates all the possible services that the user can make use of. The BIN conceptual model shows the correlations of the three planes. The correlation between user and network planes is such that the user has a number of accessible networks. The user and service plane correllation defines the services that the user have subscribed or installed. The network and service plane correlation defines the services that can be provided in the networks that the user is allowed to access.

\subsection{BIN MIB}

BIN MIB is the customer personal service palette and it should be structured according to BIN conceptual model. (Figure 6) The location of such a database can be either in BSS itself or in HBSCP's BSMP.

Actually the icons form the basis of this BIN MIB framework. They contain all the information of the service that has to be known by BSCP in order to be able to execute the service. They include for example in VOD, the BSP and its address, used picture formats and used network. On the other hand, the allowed networks are also listed with necessary parameters, e.g. transmission speed.

BIN MIB is designed to be managed with TMN (Telecommunications Management Network) architecture. An example considering UPT management can be found in /5/. 


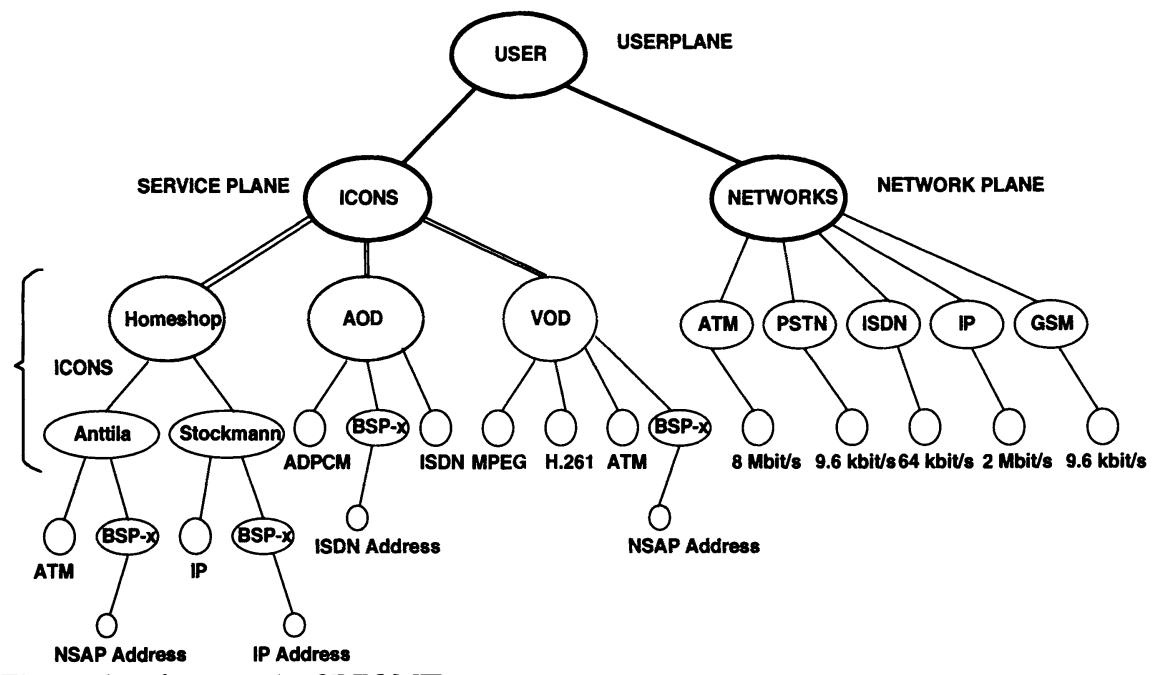

Figure 6. A framework of BIN MIB.

\subsection{TMN and BIN}

In the previous sections the Broadband IN services were discussed. The services were static services which could not be configured by the customer. The meaning of this stage was just to have a view of BIN and its possibilities. The next step is to have a remotely configurable service database where the customer could remotely configure for instance his VOD service table and get the true VOD service capabilities. The aim of this stage is to have a TMN configurable BIN service parameters. The customer's configuration would affect the BSCP database (MIB) and naturally also the BSMP, because of the charging. (Figure 7)

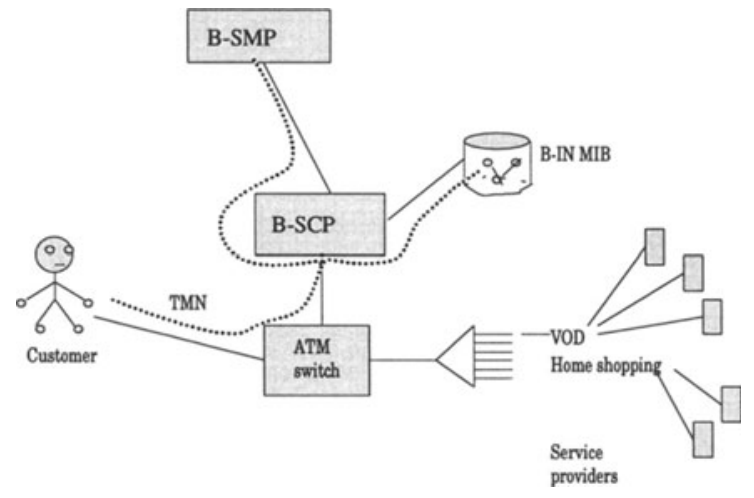

Figure 7. The use of TMN in BIN. 


\section{CONCLUSION}

A new architecture for managing Broadband Intelligent Network services has been presented. The BIN architecture is based on ATM networks which would allow also mobile station access. It makes also use of TMN architecture and QoS-related approaches, which are still under design phase.

\section{REFERENCES}

/1/ CCITT Recommendations Q.1200 series, Intelligent Network

12/ CCITT Recommendation Q.931, ISDN User-Network Interface Layer 3, Specification for Basic Call Control

13/ ITU-TS recommendation Q.2931, B-ISDN, Digital Subscriber Signalling No. 2 (DSS2), User Network Interface Layer 3, Specification for Basic Call/Connection Control

14/ K. Molin, O. Martikainen, Intelligent Network Tutorial, The Second Winterschool on Telecommunications, Helsinki, Telecom Finland, March 1994

15/ J. Airaksinen, O. Martikainen, J. Sonninen, H. Töhönen, UPT Service Management, Proceedings of the Workshop on Intelligent Networks, Lappeenranta University of Technology, 1993 